\title{
Web Inspection Using Gradient-Indexed Optics
}

\author{
John H. Sorebo, Member, IEEE, and Robert D. Lorenz, Fellow, IEEE
}

\begin{abstract}
Photoelectric sensors, used to inspect product and packaging attributes, are ubiquitous in today's manufacturing processes. Most common are single-element sensors that detect the presence or absence of an attribute, product, or package as a binary oN/OFF signal. In the web-converting industry, more detailed information is often needed of the web than a single sensor can detect. In addition, single-element sensors are often not sufficient to reliably sense product attributes. A small amount of contamination can render single-element sensors dysfunctional. They are also hindered from being able to distinguish between components on products that respond similarly to the light source but may differ in geometric shape on the product. Machine vision systems can detect much more detail and have proven effective for many challenging applications. However, due to their complexity, they are generally specified only for the most challenging or most critical quality inspections. This paper will present a new hybrid approach to product attribute sensing employing desirable aspects of both single-element sensors and machine vision systems without many of the disadvantages of each. The hybrid sensor contains a linear array of pixel elements along with a gradient-indexed lens array. The linear array allows for greater sampling and geometric characterization in one dimension while the lens array allows for very close standoff distances from the web and a 1:1 correspondence of object to image. The presentation of this sensor technology includes a tutorial on the key technical properties of gradient-indexed optics and experimental results on a composite web running on a trial machine.
\end{abstract}

Index Terms - Gradient index lens, gradient lens array, hybrid sensor, linear array, machine vision system, web sensing.

\section{INTRODUCTION}

$\mathbf{I}$ NCREASINGLY, consumer-product-converting businesses are relying on higher web speeds and improved web process controls to raise operating profits. This strategy is being executed at the same time as products are becoming more complex to fulfill heightened consumer aesthetic and performance expectations. One area of technical challenge seen as a result of these changes is the registration and inspection of product components. Registration is a control mechanism in which product components are aligned in such a way as to produce a finished product with the targeted dimensions. Alignment of

Paper PID-05-15, presented at the 2005 IEEE Pulp and Paper Industry Conference, Jacksonville, FL, June 19-23, and approved for publication in the IEEE TRANSACTIONS ON INDUSTRY APPLICATIONS by the Pulp and Paper Industry Committee of the IEEE Industry Applications Society. Manuscript submitted for review June 23, 2005 and released for publication September 1, 2005. This work was supported by Kimberly-Clark Corporation and by the Wisconsin Electric Machines and Power Electronics Consortium (WEMPEC) of the University of Wisconsin, Madison.

J. H. Sorebo is with Adult/Feminine Care, Kimberly-Clark Corporation, Neenah, WI 54956 USA (e-mail: jsorebo@kcc.com).

R. D. Lorenz is with the Departments of Mechanical Engineering and Electronics and Communications Engineering, University of Wisconsin, Madison, WI 53706 USA (e-mail: lorenz@engr.wisc.edu).

Digital Object Identifier 10.1109/TIA.2005.858275 product components is absolutely crucial in consumer product converting. Improper alignment of webs can cause problems such as slitting a product to the wrong width, spraying adhesive off the edges of a web, or failing to register components to their targeted dimensions. Although there are many facets of successful web registration, one critical element is the ability of sensors to accurately sense the edges of a web and product components in a timely manner.

In general, web registration and inspection control is limited to two dimensions. These dimensions are the direction of the web travel called the machine direction (MD) and the perpendicular cross direction (CD). Whereas MD registration control is typically accomplished using only per product sensor information, $\mathrm{CD}$ registration control often requires continuous sensor information. This dichotomy has traditionally given rise to sensors designed exclusively for MD or CD control. Small single-element sensors and machine vision systems have been used as feedback devices for MD registration while large single-detector or unbuffered line scan array technology has been used for $\mathrm{CD}$ registration. (Most digital camera systems use a two-dimensional (2-D) pixel array. Line scan detectors feature a one-dimensional (1-D) linear pixel array of photo elements.)

\section{A. MD Sensor Conventions}

MD sensors accomplish a variety of discrete presence inspections from splice tape detection to component edge and product case detection. Because single-element sensors have one detector, they can be extremely fast and still operate with very low light contrast levels. They are produced inexpensively in a variety of different form factors and sizes and are easy to install and setup. Recently, configurable delays and signal processing techniques have been employed to filter out signal noise. It is for these reasons that the most common type of sensor used in the converting industry today are small singleelement sensors. But these sensors are not adequate for all situations. A large category of presence and measurement inspection requires more than just a single piece of information. For this category of inspection, machine vision systems have provided a technical solution.

Machine vision systems have the luxury of having much more information available to them prior to making a presence or measurement decision. In the case of 2-D camera systems, a digital image is taken of the camera's field of view, which is the area of the product or a certain part of the product. Line scan camera systems assemble an image by scanning the product and buffering the data as the web traverses the machine. In either case, tools are configured to make the necessary calculations of the product attributes and information is fed back to a control system in a variety of ways. 


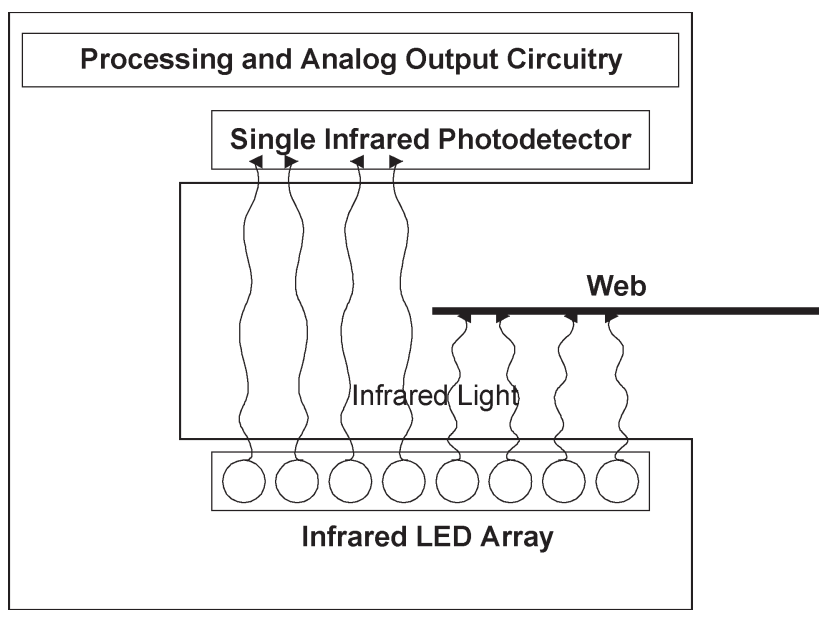

Fig. 1. Conventional $\mathrm{CD}$ web edge sensor configuration.

\section{B. CD Sensor Conventions}

In the case of CD sensing, edge sensors that are most often used in the converting industry are based on the transmission of infrared light from light-emitting diodes (LEDs) across an open air gap that is partially obstructed by the web edge in question. On the other side of the web lies a single photo detector, which receives the light and produces a number of electron-hole pairs in the semiconductor proportional to the intensity of the light it has received within the wavelength band to which the semiconductor is responsive. The electron-hole pairs form an electrical potential, which is read by the photo detector interface circuitry as an analog voltage. The analog voltage is buffered and sent to a current or voltage output driver circuit. A web control processor then reads this signal. The output level, in the form of a current source, is a nonlinear function of the lateral position of the web, the material opacity (optical transmittance), and any other spatial property that could modulate the light energy impinging on the photo detector. Fig. 1 shows this conventional $\mathrm{CD}$ web edge sensing configuration.

Line scan detector array technology has also been used successfully in determining the location of $\mathrm{CD}$ web edges for converting webs. A line scan array effectively samples the light intensity distribution in a direction orthogonal to the edge of the web. The resulting sampled image can then be processed by image processing techniques to determine the relative edge location with respect to a reference. Line scan arrays are generally less sensitive to web opacity variations because more spatial information is available to the sensor. Most line scan arraybased sensors are designed into a camera-style format, where a spherical lens system functions to collect light and focuses it on the linear array. Although camera-style implementations of line scan arrays allow for off-the-shelf application, they do have some limitations. One limitation is the working distance (distance from lens to object) required. Line scan arrays would find wider acceptance if they could be placed in a very confined area, where distances from objects to line scan array are only on the order of millimeters, not feet or inches as is the case with standard spherical lens systems. Another limitation of the camera-style format is in the establishment of field of views and its impact on pixel length calibration, i.e., pixel

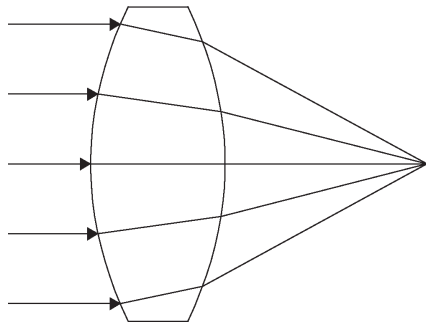

Fig. 2. Conventional spherical lens collecting light from a distant source and focusing it to a single point by means of refraction only at the surface of the lens.

resolution. There is often a tradeoff between getting enough pixel resolution by zooming in versus having enough field of view. Zooming in to improve pixel resolution also means that absolute pixel resolution is not clearly defined and thus a calibration procedure must be used.

\section{Gradient Lens Array (GLA) Sensor Introduction}

Standoff and pixel length calibration and resolution issues would not be critical if the line scan detector array used GLA optics. With GLA optics, the field of view is a one-to-one relationship with the linear array due to unity magnification, and the focal distance is on the order of millimeters, not feet or even inches. This means that a very compact sensor can be designed to have the full functionality of a camera-style sensor with no setup calibrations required. And since the optics are linear, GLAs can be made to fit any length of image array without suffering from lack of resolution or large object to lens distances. Commercial GLAs have become readily available [2].

The current focus of machine vision and sensor manufacturers is to develop MD inspection systems that have the simplicity advantages of a discrete sensor with the capability of a machine vision system. This is known in industry as a hybrid or vision sensor. CD inspection largely continues to be a specialization of those manufacturers who design complete web guiding solutions. The purpose of this paper is to discuss a web sensor design that can provide some of the capabilities of a machine vision system and a $C D$ sensor with the simplicity of a singleelement discrete sensor.

\section{GLA SENSOR PRoperties}

A gradient index lens has an index of refraction gradient in the radial direction of the lens optical material. The index of refraction is highest in the center of the lens and decreases with radial distance from the axis according to [2]

$$
N(r)=N_{0}\left(1-\frac{A}{2} r^{2}\right)
$$

where $N_{0}$ is the index of refraction at the lens axis, $A$ is a gradient constant, and $r$ is the radius from the lens axis. The parabolic index profile allows the lens to focus light in a shorter distance than a conventional spherical lens, which can refract light only at its surfaces. Figs. 2 and 3 depict these differences.

The spatial refraction index gradient property of the gradient index lens allows it to take on a cylindrical format with flat input 


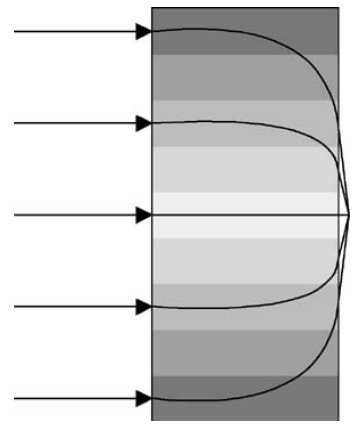

Fig. 3. Gradient index lens collecting light from a distant source and focusing it to a single point by means of refraction with a radially varying index lens material.

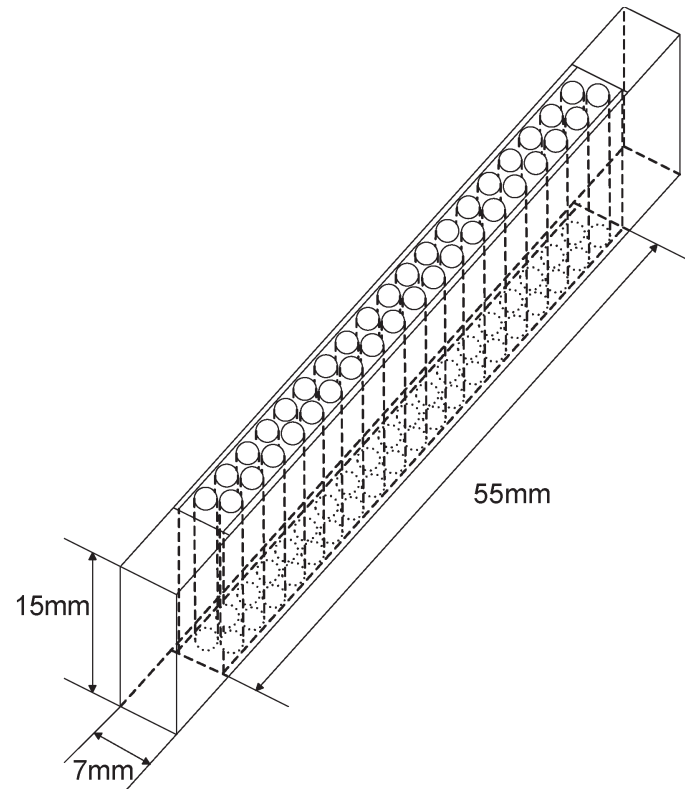

Fig. 4. GLA with two rows of lenses as used for the experimental setup discussed in this paper.

and output surfaces. This cylindrical format lends itself to many applications because of the ease in packaging multiple lenses in a parallel structure. This parallel lens structure can be fabricated in 1-D and 2-D arrays, whereby images from adjacent lenses overlap and form a continuous erect image (i.e., not inverted). This type of GLA serves the function of being able to focus scattered light onto a line scan charge-coupled device (CCD) or CMOS array. An example of such a GLA is shown in Fig. 4.

Working distance is often a critical factor in web sensing because of other operations that need to be performed in the space above the web. For evaluation of working distances, spherical lens systems are known to require a working distance roughly given by

$$
W_{d}=F_{d}\left(\frac{F_{v}}{L_{a}}+1\right)
$$

where

$F_{d} \quad$ focal distance of the lens;

$F_{v} \quad$ field of view;

$L_{a}$ array length.

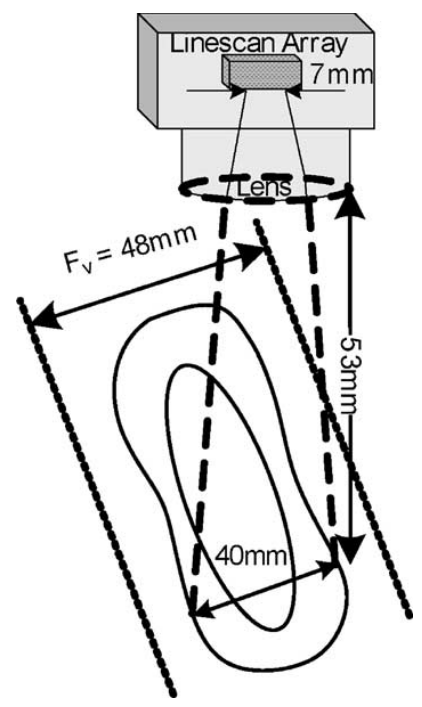

Fig. 5. Conceptual representation of a line scan camera using conventional optics inspecting a web composite.

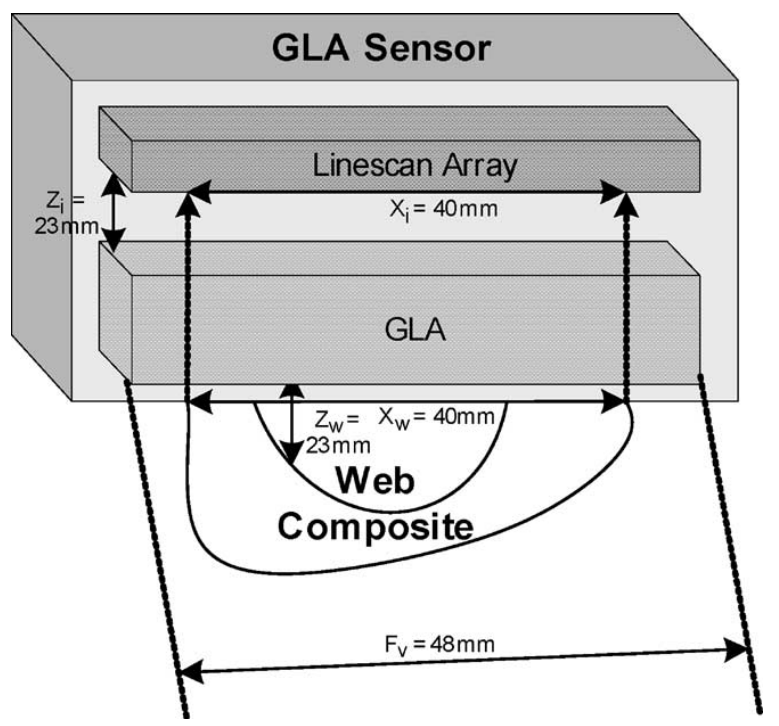

Fig. 6. Conceptual representation of a GLA-based line scan sensor inspecting a web composite.

As an example, a web edge sensor consistent with the application presented in this paper might require a field of view of $48 \mathrm{~mm}$. A 1/3-in 1-D CCD line scan array has a length of about $8.5 \mathrm{~mm}$. An objective lens with a focal distance of less than $8 \mathrm{~mm}$ is generally unwise due to the large curvature of the lens. For this example, a spherical lens would require a working distance of $53 \mathrm{~mm}$, as shown conceptually in Fig. 5.

It should also be noted that for conventional spherical optics, the object and image lengths are not equal. Thus, a calibration procedure must be performed to accurately translate system pixel data to actual product measurements.

These working distance and calibration issues with spherical lenses can be resolved by using GLA-based line scan sensors shown conceptually in Fig. 6. 


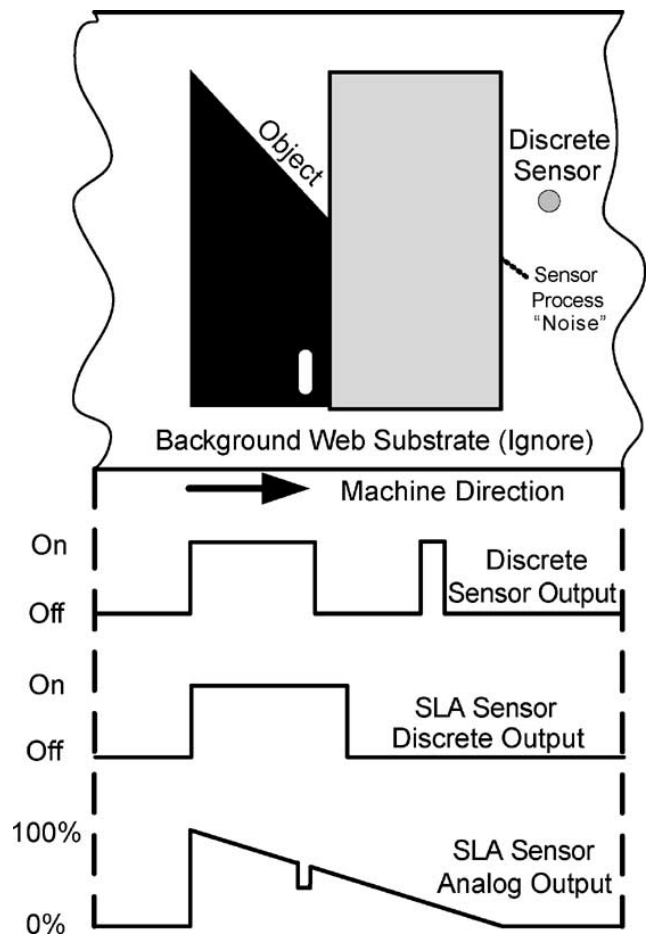

Fig. 7. Comparison of the filtering property of a GLA sensor with a singleelement discrete sensor.

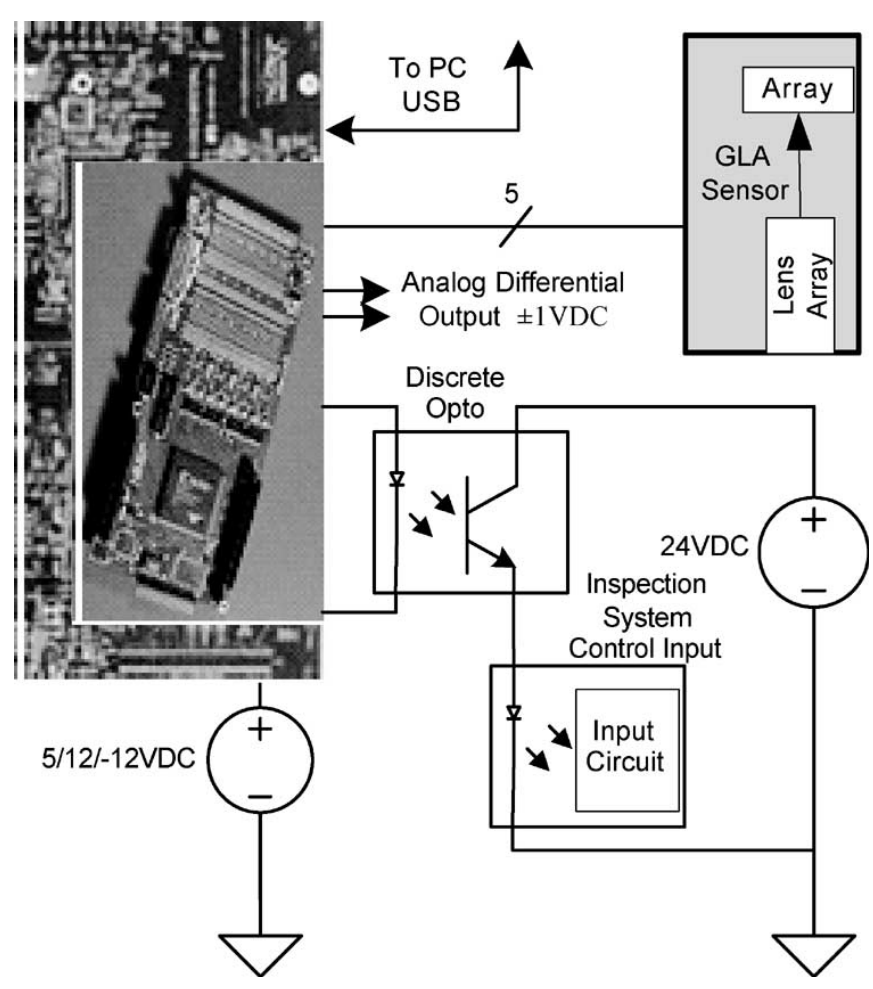

Fig. 8. Hardware elements of the GLA sensor prototype.

Working distances achieved with GLAs are on the order of 4-30 mm. Furthermore, there is a one-to-one correlation of the line scan pixel data to the product, without a need for additional calibration procedures. In effect, a GLA-based sensor can provide machine vision information but with virtually the

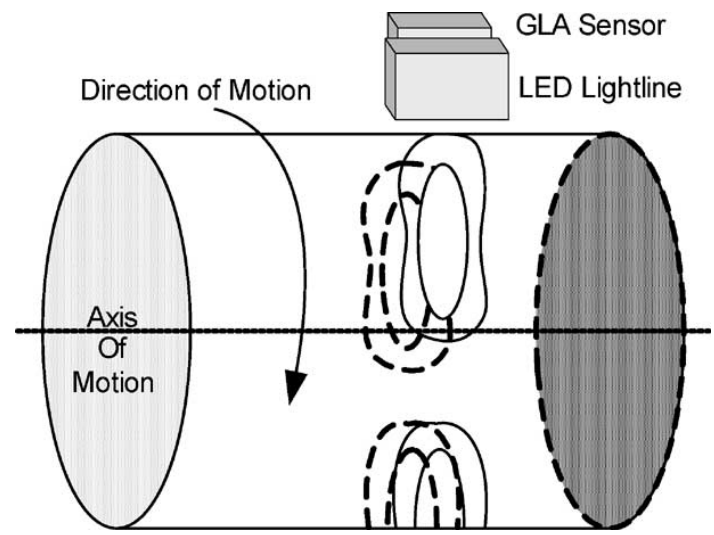

Fig. 9. Experimental setup showing the three feminine pads placed on the clear drum with a GLA sensor and a red LED light line light source mounted to the drum frame via a micrometer stage.

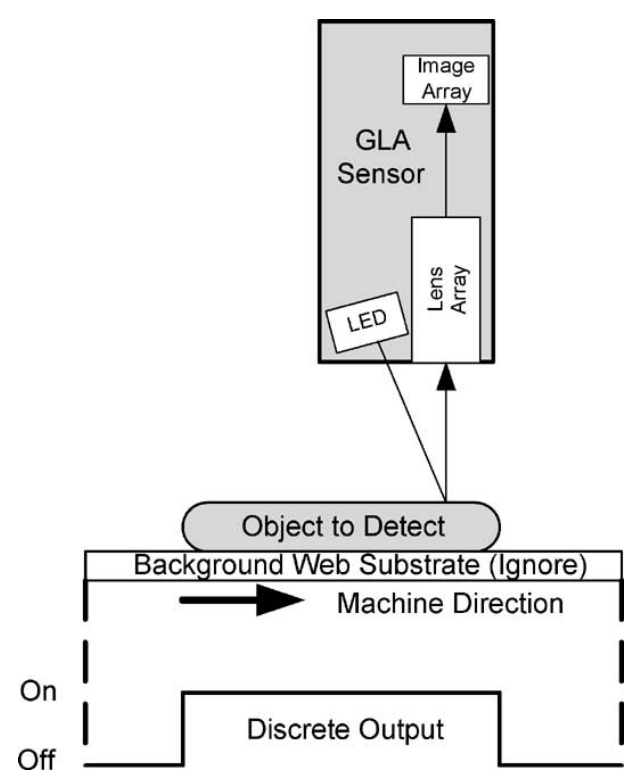

Fig. 10. Cross-sectional view of a GLA sensor with an object-to-lens array distance of $23 \mathrm{~mm}$ was inspecting a web component (pad) on top of the substrate (drum)

same form factor and ease of setup of a discrete single-element sensor.

Fig. 7 demonstrates the usefulness of such a sensor. If an application required a sensor to detect the presence and $\mathrm{CD}$ edge location of an object (in this case, a triangular piece), on top of a web substrate, a standard CD-type sensor would not likely work because cut and place operations are often done using vacuum conveyors to ensure the web composite is controlled. Therefore, both top and bottom of the object to be detected would not be exposed. A discrete sensor often times fails to detect the correct web object edges due to contaminants in the process in the figure as process "noise."

The GLA sensor depicted in Fig. 7 is able to span the entire width of the object. When it comes across the contaminant, it notices by pixel counting that the contaminant is not large enough to constitute the object edge for which it is searching. 


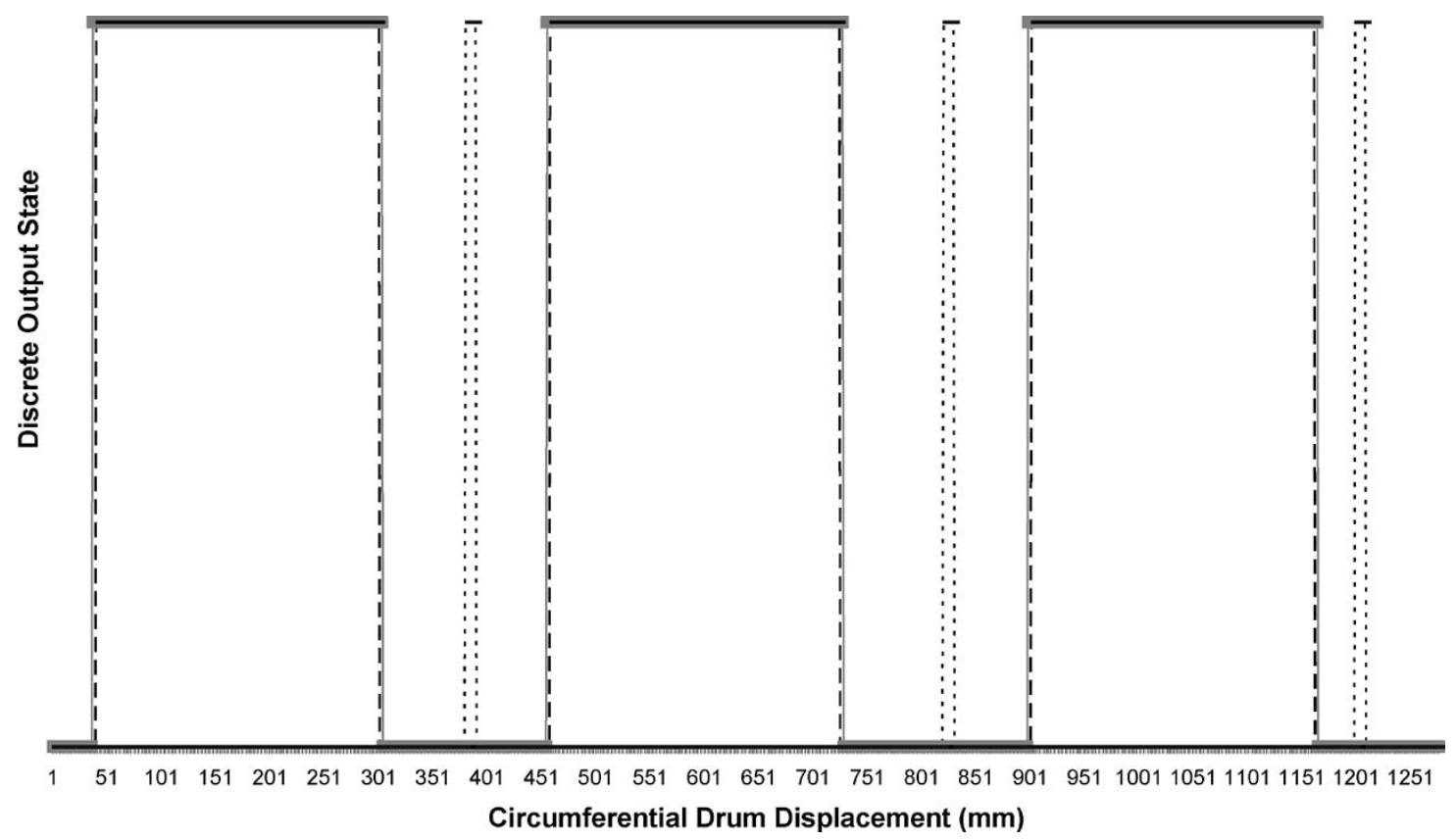

Fig. 11. Plot of discrete output state versus circumferential drum displacement for three test conditions. Legend: — test with no contamination; --- test with pad contamination with the pixel threshold set to 200 pixels $(635 \mu \mathrm{m})$; and ... test with pad contamination filtered out by the sensor.

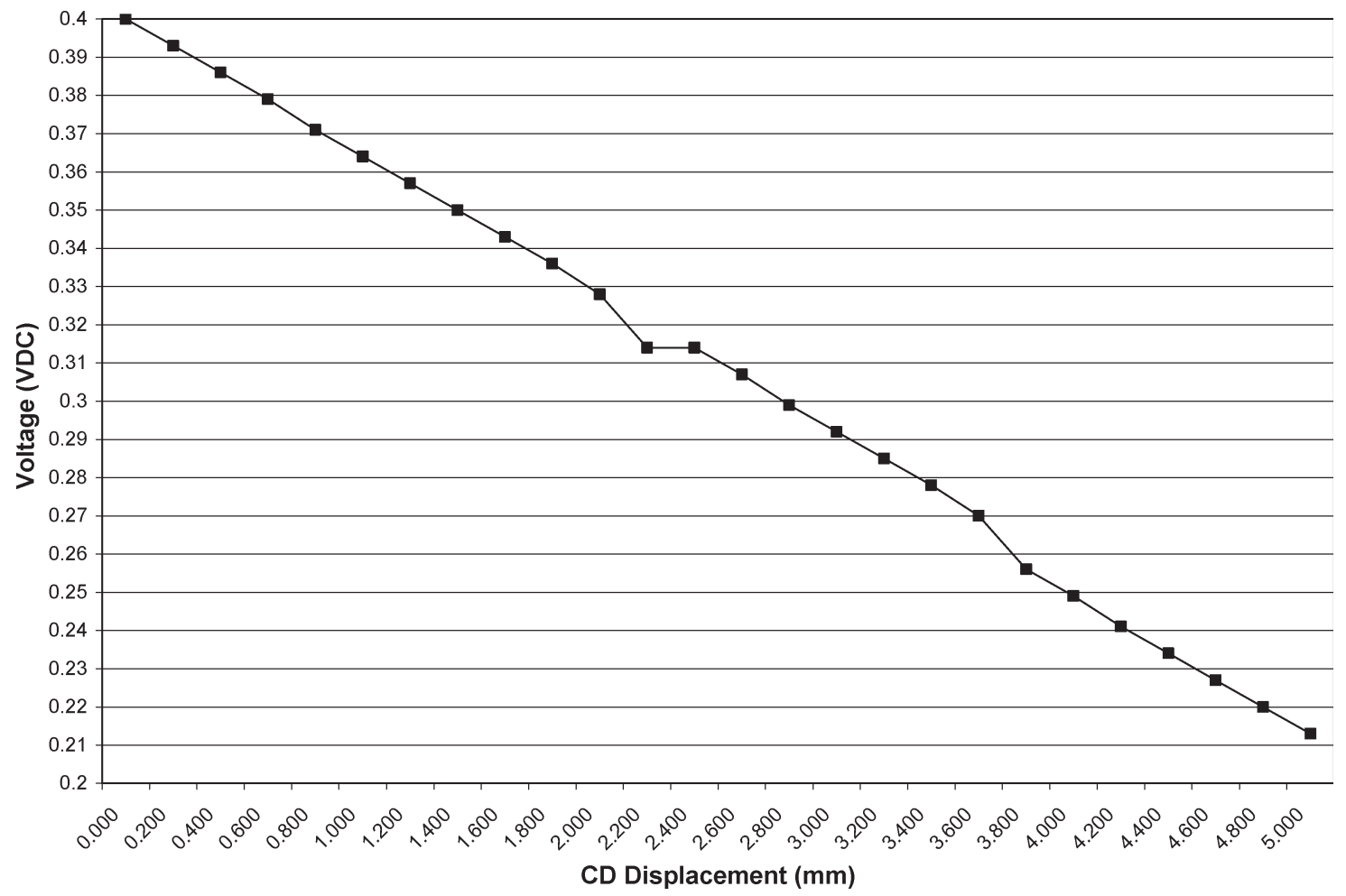

Fig. 12. Plot of analog output versus CD displacement whereby the 5-mm incremental displacement was achieved by micrometer stage movement of the sensor with respect to the pad.

It also filters out the hole in the object because it is also not large enough to diminish the pixel count below the threshold. This filtering property makes the GLA sensor inherently more robust than a single point sensor. The fact that a GLA sensor can extract high-quality information with just a single line scan is also a significant advantage when compared to camera systems with 2-D scanning or with buffered line scanning to create a 2-D image. In these cases, the time to extract a high-quality decision is significantly reduced with the GLA sensor.

\section{EXPERIMENTAL SENSOR System}

The experimental objective was to evaluate the use of the GLA properties as part of a multipixel line scan sensor system 
that would function as both an MD sensor (discrete output) and a CD sensor (analog output) simultaneously. The sensor would simply act as a pixel counter by comparing the number of pixels that received a light level above a configurable threshold. Based on projected machine speeds, the sensor would have to update its discrete and analog output in less than $1 \mathrm{~ms}$. To ensure longevity and a small form factor of the light source, LED lighting was required. Fig. 8 shows the hardware used in the sensor system design.

The 1-ms specification for the outputs is one of the critical paths in sensor design. A linear array was selected with a 48-mm line length. The 768-pixel line scan array scans in less than $1 \mathrm{~ms}$ while the two taps are operated in parallel.

To accomplish the processing for this, a digital signal processor (DSP) board was used in conjunction with a fieldprogrammable gate array (FPGA) analog daughter board. The DSP board provided a universal serial bus (USB) interface to the PC and allowed the configuration of the line scan rate and the pixel light and number thresholds used in the FPGA's pixel counting algorithm. The FPGA board interfaces directly to the line scan array in the sensor head. It provides DC power, a clock, a synchronization start bit, and a common ground for the array. The array feeds back analog pixel information to the FPGA board. The FPGA board in turn converts the analog voltages into an 8-bit digital representation and copies the raw data to the DSP board via a common External Memory InterFace (EMIF) bus for line scan data recording on the PC. In this way, the DSP board acts as a PC interface and the FPGA board as the real-time processor. A high-speed optocoupler isolates the sensor's processors from the external machine control system I/O circuitry.

\section{EXPERIMENTAL TEST STAND}

Fig. 9 shows a sketch of the experimental setup that was used to emulate the properties of a continuous web of converted products. Fig. 10 shows the same setup from a crosssectional standpoint in which the sensor system components are called out explicitly. Three ultrathin feminine pads were placed on a clear drum of 1276-mm circumference. The pads had lengths of 272, 272, and $274 \mathrm{~mm}$, respectively. Three separate tests were performed to evaluate the capabilities of the GLA sensor.

1) The drum was rotated at a speed that emulated a web surface speed of $1000 \mathrm{ft} / \mathrm{min}$. The sensor's discrete output was recorded, indicating the presence of the pad underneath the sensor. The signal lengths were compared against the offline measured product.

2) The drum was again rotated at a speed that emulated a web surface speed of $1000 \mathrm{ft} / \mathrm{min}$. Contamination in the form of $1-\mathrm{cm}^{2}$ pieces of the pad were placed between each pad (three instances altogether). The intent here was to show the sensor system's robustness to contamination.

3) With the drum at rest, the GLA sensor system's analog output was recorded as the sensor was moved $5 \mathrm{~mm}$ with a micrometer in the cross direction (CD) in $200-\mu \mathrm{m}$ intervals. This test was performed to evaluate the linearity that can be achieved by the sensor system in $\mathrm{CD}$ application.

\section{EXPERIMENTAL RESUlts}

The first tests evaluated the noise filtering properties of the GLA sensor when used as a discrete output (ON/OFF) device. Fig. 11 shows these results under three test conditions. It can be seen that the GLA sensor accurately determines the presence of the pad while filtering out pad contamination. The pad lengths recorded by the sensor were within the tolerance of the test apparatus $( \pm 5 \mathrm{~mm})$ when compared with the offline measured length.

Results of the third test are shown in Fig. 12. The sensor demonstrated a high degree of linearity in making analog CD measurements.

\section{CONCLUSION}

The experimental results show that a gradient lens array (GLA) sensor has the potential to be used for web inspection applications, where single-detector discrete output (ON/OFF) sensors are not adequate for differentiating noise produced from real-world web components. The GLA sensor is a suitable alternative to machine vision systems that would normally be necessary. GLA sensors have similar properties to singleelement sensors in that they are close enough to the webs to allow static electricity and contaminants to build up on their lenses. However, they do not require the amount of machine space, setup procedures, and programming that machine vision systems do and can potentially be deployed at lower overall cost.

Work has been done to evaluate the performance advantages of the GLA sensor, but much work remains to be done in the areas of signal processing and system design to achieve robust and cost-effective utilization in web-converting processes.

\section{REFERENCES}

[1] J. H. Sorebo and R. D. Lorenz, "Web detection with gradient-indexed optics," U.S. Patent US2003/0116725, Jun. 26, 2003.

[2] SELFOC Lens Array Technical Notes, NSG America, Somerset, NJ, Jun. 1996.

[3] Y. Ying-hai, J. Wei-tong, Y. Ben-li, L. Zu-ning, and L. Peng, "Imaging properties and measurement of SELFOC lens and SLA," in Proc. IEEE Int. Conf. Industrial Technology, Guangzhou, China, Dec. 5-9, 1994, pp. $422-425$.

[4] J. Goodman, Introduction to Fourier Optics, 2nd ed. New York: McGrawHill, 1996

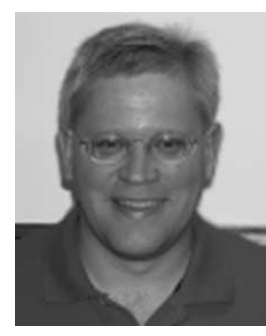

John H. Sorebo (S'98-M'00) received the B.S.E.E degree from the University of Minnesota, Twin Cities, in 1994, and the M.S.E.E. degree in electrical engineering from the University of Wisconsin, Madison, through the university's Engineering Outreach Program in 2000

$\mathrm{He}$ is currently an Electrical Engineer with Kimberly-Clark Corporation, Neenah, WI. In his 11 years with the company, he has worked in plant operations and on multiple converting process design teams. His current area of emphasis is in the area of high-speed product registration and inspection for converting machines. 


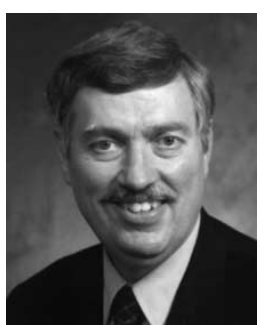

Robert D. Lorenz (S'83-M'84-SM'91-F'98) received the B.S., M.S., and Ph.D. degrees from the University of Wisconsin, Madison, in 1969, 1970, and 1984, respectively, and the M.B.A. degree from the University of Rochester, Rochester, NY, in 1980.

From 1972 to 1982 , he was a Member of the Research Staff at Gleason Works, Rochester, NY, working principally on high-performance drives and synchronized motion control. Since 1984, he has been a Member of the Faculty at the University of Wisconsin, Madison, where he is the Mead Witter Foundation Consolidated Papers Professor of Controls Engineering in both the Department of Mechanical Engineering and the Department of Electrical and Computer Engineering. He was a Visiting Research Professor at the Electrical Drives Group, Catholic University of Leuven, Leuven, Belgium, in the summer of 1989, and in the Power Electronics and Electrical Drives Institute, Technical University of Aachen, Aachen, Germany, in the summers of 1987, 1991, 1995, 1997 and 1999, respectively, and was the SEW Eurodrive Guest Professor from September 1, 2000 to July 7, 2001. He is the Co-Director of the Wisconsin Electric Machines and Power Electronics Consortium, which will celebrate its 25th anniversary in 2006. It is the largest industrial research consortium on motor drives and power electronics in the world. He is also the Thrust Leader for control and sensor integration in the Center for Power Electronic Systems, a National Science Foundation (NSF) Engineering Research Center that is a joint ERC with the Virginia Polytechnic Institute, Rensselaer Polytechnic Institute, University of Puerto Rico-Mayaguez, and North Carolina A\&T. His current research interests include sensorless electromagnetic motor/actuator technologies, real-time signal processing and estimation techniques, precision multi-axis motion control, and ac/dc drive and high-precision machine control technologies. He has authored more than 170 published technical papers and is the holder of 23 patents with three more pending.

Dr. Lorenz is a member of the American Society of Mechanical Engineers (ASME), the Instrument Society of America (ISA), and The International Society for Optical Engineers (SPIE). He is also a member of the IEEE Sensor Council AdCom. He was a Distinguished Lecturer of the IEEE Industry Applications Society (IAS) for 2000/2001, the IEEE Industry Applications Society (IAS) President for 2001, past Chair of the IAS Awards Department, past Chairman of the IAS Industrial Drives Committee, is an IEEE Division II Director for 2005/2006, and is a Member of the IAS Industrial Drives Committee, Electrical Machines Committee, Industrial Power Converter Committee, and Industrial Automation and Control Committee. He is the immediate past Chair of the Periodical Committee and the current Chair of the Periodicals Review Committee for the IEEE Technical Activities Board. He was awarded the 2003 IEEE IAS Outstanding Achievement award, which honors his outstanding contributions and technological developments in the application of electricity to industry. He has won 18 prize paper awards. He is a Registered Professional Engineer in the States of New York and Wisconsin. 